\title{
Drug firms must cut price of pneumonia vaccine, says MSF
}

In this News story about the cost of the pneumonia vaccine (BMJ 2015;350:h2238, doi:10.1136/bmj.h2238), we said that the lowest price was around $\$ 10$ a dose. The lowest price is actually around $\$ 10$ per child, which is for all three doses; per dose the cost is around \$3.30-\$3.40. We apologise for this error.
Cite this as: BMJ 2015;350:h2289

๑ BMJ Publishing Group Ltd 2015 